\title{
Stress phase angle regulates differentiation of human adipose-derived stem cells toward endothelial phenotype
}

\author{
Shahrokh Shojaei ${ }^{1} \cdot$ Mohammad Tafazzoli-Shadpour $^{2} \cdot$ Mohammad Ali Shokrgozar $^{3} \cdot$ Nooshin Haghighipour $^{3}$. \\ Fatemeh Hejazi Jahromi ${ }^{4}$
}

Received: 16 February 2018 / Accepted: 6 May 2018 / Published online: 21 May 2018

(c) The Author(s) 2018

\begin{abstract}
Endothelial cells are subjected to cyclic shear by pulsatile blood flow and pressures due to circumferential stresses. Although most of the researches on this topic have considered the effects of these two biomechanical forces separately or concurrently, few studies have noticed the interaction of these cyclic loadings on endothelial behavior. Negative temporal stress phase angle, defined by the phase lag between cyclic shear and tensile stresses, is an established parameter which is known to have substantial effects on blood vessel remodeling and progression of some serious cardiovascular diseases. In this research, intermittent shear and tensile stresses with different stress phase angle values were applied on human adipose stem cells (ASC). The expression level of three major endothelial-specific genes, elastic modulus of cells and cytoskeleton actin structure of cells were studied and compared among control and three test groups subjected to stress phase angle values at $0^{\circ},-45^{\circ}$, and $-90^{\circ}$. Mechanical properties of ASCs were determined by atomic force microscopy and actin fiber structure was visualized by confocal imaging through Phalloidin staining. Results described a decrease in expression of FLK-1 and VE-cadherin and rise of vWF marker expression in case of higher negative stress phase angles. The Young's moduli of cells were significantly higher and cytoskeletal actin structure was more organized with higher thickness for all test samples subjected to combined stresses; however, these features were less magnificent for applied stress phase angles with higher negative values. The results confirmed significant effects of SPA on endothelial differentiation of mesenchymal stem cells.
\end{abstract}

Keywords Stress phase angel $\cdot$ Shear stress $\cdot$ Cyclic stretch $\cdot$ Adipose mesenchymal stem cells $\cdot$ Cell behavior

Shahrokh Shojaei

shahrokh.shojaei@gmail.com

$\triangle$ Mohammad Tafazzoli-Shadpour

Tafazoli@aut.ac.ir

1 Faculty of Biomedical Engineering, Central Tehran Branch, Islamic Azad University, 13185/768, Tehran, Iran

2 Cardiovascular Engineering Lab., Faculty of Biomedical Engineering, Amirkabir University of Technology, Tehran 158754413, Iran

3 National Cell Bank of Iran, Pasteur Institute of Iran, Tehran 1316943551, Iran

4 Hard Tissue Engineering Research Center, Tissue Engineering and Regenerative Medicine Institute, Central Tehran Branch, Islamic Azad University, 13185/768, Tehran, Iran

\section{Introduction}

Endothelial cells (ECs) cover the entire cardiovascular bed and role as the blood-vessel wall interface within arteries. ECs are exposed to circumferential stress which is caused by pressure pulse acting on elastic vessel walls. Shear stress is another vital biomechanical stimulus applied on ECs due to the pulsatile blood flow. There is sufficient in vitro evidence that may imply to possible influence these two mechanical stresses have on many essential traits of both ECs and stem cells. For instance, it was demonstrated that shear stress affects gene expression (Paul et al. 2018), morphology and adhesion (Charrier et al. 2018; Paim et al. 2018), extracellular matrix secretion, mechanical properties, and angiogenesis ability (Charoenpanich et al. 2017; La and Tranquillo 2018; Vining and Mooney 2017) of ECs and stem cells. Some of the most significant biological characteristics of ECs can be regulated by application of pulsatile shear stress (Dolan et al. 2011); shear stress out of biological range values contribute 
to conditions in which vascular diseases develop such as activation of IGF-1 (Elhadj et al. 2003), uptake of LDL (Kang et al. 2014), and pathogenic remodeling (Dolan et al. 2011). Cyclic tension has also been indicated to be influential on endothelial behavior (Barron et al. 2007; Haghighipour et al. 2010). Cyclic stretch promotes smooth muscle genes in stem cells, while it attenuates endothelial specific genes among ECs (Haga et al. 2007). In addition, the substrate's characteristics have distinct influence on morphology, mechanical properties, and other important characteristics of the cultured cells (Jeon et al. 2014).

Although these biomechanical stimuli are essential in regulation of vital biological characteristics of ECs, the interaction between these biomechanical forces has attracted less attention. There are some clues which illustrate well the dominant effects of interaction between tensile and shear stresses on endothelial behavior. While many earlier studies have stressed the influence of altered pattern of shear stress on atherogenesis (Bassiouny et al. 1992; Ku et al. 1985), some other investigations have challenged the sole role of shear stress pattern in situations such as intimal thickening in the right coronary (Joshi et al. 2004) and carotid bifurcation (Steinman et al. 2002). In general, the interaction between shear and tensile stresses has been widely suggested to play an important role on endothelial behavior (Joshi et al. 2004; Steinman et al. 2002).

The phase lag between wall shear stress (WSS) and circumferential stress (CS) within arteries, known as the stress phase angle (SPA), is a primary parameter that characterizes the interaction between these two stresses. This factor could be derived based on the phase difference between first harmonics of WSS and CS waves. It has been shown that large negative values of SPA are correlated with the regions which are vulnerable to endothelial injury and plaque formation (Tada and Tarbell 2005). Further investigations have suggested that large negative values of SPA strengthen the risk of atherosclerosis and intimal hyperplasia (Dancu and Tarbell 2006; Qiu and Tarbell 2000b). It has been documented that large negative SPA attenuates the expression of some vasodilator factors including nitric oxide (NO) and prostacyclin (Dancu and Tarbell 2006) and inhibits the production of some vasoconstrictor agents such as endothelin-1 (Qiu and Tarbell 2000a).

Since mature endothelial cells within the cardiovasculature are continuously exposed to concurrent shear and tensile forces, it has been suggested that application of such mechanical stimuli may enhance differentiation of mesenchymal stem cells toward functional endothelial phenotype (Maul et al. 2011). Previously, we reported that applying shear stress alone or combined with cyclic stretch on adipose derived stem cells (ASCs) could lead to enhanced expression of endothelial-specific genes and some other endothelial vital traits in ASCs, while the cyclic stretch alone did not improve those expressions (Shoajei et al. 2014). A same trend in elevation of expression of endothelial genes has been reported when endothelial cells are exposed to different patterns of shear stress (Yamamoto et al. 2003).

On the other hand, reduction of endothelial genes was observed when endothelial cells were subjected to cyclic stretch alone, whereas smooth muscle cell markers were intensified; such variation in gene expression is pathologically regarded as inauguration of atherosclerosis (Cevallos et al. 2006). Effects of concurrent shear and tensile loadings on endothelial and mesenchymal stem cell behavior have also been investigated (Owatverot et al. 2005; Shoajei et al. 2014). In our previous research works, we addressed the effect of in-phase $(\mathrm{SPA}=0)$ shear stress and tensile stretch on gene expression, mechanical properties of the membrane, angiogenesis ability, and other vital characteristics of ASCs and endometrial stem cells (Shojaei et al. 2013a, b, c, 2015). Although there are relatively high number of studies on concurrent shear stress and tensile stretch influencing the differentiation and transdifferentiation of stem cells, the effects of interaction between cyclic shear and tensile stresses are not assessed widely and perfectly on endothelial differentiation of stem cells. To our best knowledge, this is the first time that the effects of the SPA have been investigated on gene expression, mechanical properties, and actin fibers' organization and arrangement. Here, we hypothesized that SPA, as an indicator of such interaction, is effective in differentiation of mesenchymal stem cells toward endothelial fate.

\section{Materials and methods}

The SPA was applied by the phase lag between two cyclic stresses with harmonic profiles. Cells without any treatment were set as control samples, while they were cultured in the same conditions as the test samples. Samples from three test groups with different SPA values were exposed to mechanical loading using custom-made apparatus, and at least three tests were carried out for each test group. Expression of endothelial specific genes, cell mechanical properties, and cytoskeletal structure was analyzed to evaluate the influence of SPA on differentiation toward endothelial phenotype. The mean \pm SD values of elastic modulus of cells and gene expression levels of three endothelial specific genes were calculated for control and test groups. Statistical $t$ test paired analysis was performed between control group and each test group to investigate differences among gene expression levels and elastic moduli of samples and the significant difference was set as $P<0.05$. In addition, multifactorial ANOVA analysis was carried out with the same significant difference to compare all the tests and control groups. 


\section{Cell preparation}

ASCs were extracted according to published protocols with some minor modifications (Estes et al. 2010). Pre-umbilical fat tissues were provided from healthy female donors between 30 and 35 years, during abdominal surgery with informed consent. Fat tissues were cut into small pieces and transferred to collagenase type I solution of $0.1 \mathrm{~g}$ collagenase (invitrogen, USA) in $100 \mathrm{~mL}$ phosphate buffer saline (Sigma, USA). The solution was incubated at $37^{\circ} \mathrm{C}$, $5 \% \mathrm{CO}_{2}$, and $95 \%$ relative humidity for $45 \mathrm{~min}$, while it was shaken every $5 \mathrm{~min}$, followed by removal of undigested parts. The culture medium included Dulbecco's modified Eagle's medium (DMEM, Gibco, USA) with $15 \%$ fetal bovine serum (Gibco, USA). The solution was centrifuged for $5 \mathrm{~min}$ at $2000 \mathrm{rpm}$. Then, the supernatant was removed and the bottom pellet containing ASCs and red blood cells were dissolved in fresh culture medium. The resultant solutions were transferred to a cell culture flask (T-25) and after an overnight RBCs were easily detached by light pipetting.

\section{Cell characterization}

The cells were characterized using flow cytometry assay by assessing two specific markers for mesenchymal stem cells (CD44, CD90) and one for hematopoietic stem cells (CD45). Briefly, $35 \times 10^{3}$ cells were counted and suspended in micro-centrifuge tube containing $90 \mu \mathrm{L}$ serum-PBS (5\% FBS in PBS). Then, $10 \mu \mathrm{L}$ antibody/isotype was added and the tube was shaken for $1 \mathrm{~h}$ while protecting from light. The micro-tube was centrifuged ( $800 \mathrm{rcf}, 5 \mathrm{~min}$ ) and the supernatant was removed; then, the pellet was resuspended in serum-PBS $(500 \mu \mathrm{L})$. After three times washing, the flow cytometry assay was conducted by forward scatter and sidescatter analyses.

\section{Multipotential differentiation ability}

Multipotential differentiation ability of ASCs was analyzed through adipogenic and osteogenic differentiations. Cells from passage 3 were treated by adipogenic medium containing DMEM, 10\% FBS, $0.5 \mu \mathrm{M}$ IBMX (3-isobutyl1-methylxanthine), $1 \mu \mathrm{M}$ dexamethasone, $10 \mu \mathrm{g} / \mathrm{mL}$ insulin, and $100 \mu \mathrm{M}$ indomethacin Sigma, (USA) for 3 weeks. The medium was changed every 3 days; after then, the cells were fixed by $4 \%$ paraformaldehyde (Sigma, USA) and washed in $50 \%$ isopropanol. Lipid vacuoles were visible by oil red staining. For osteogenic differentiation, ASCs were exposed to osteogenic differentiating medium (DMEM, $10 \%$ FBS, $100 \mathrm{nM}$ dexamethasone, $10 \mathrm{mM}$ beta-glycerol phosphate and L-ascorbic acid 2-phosphate, Sigma, USA) for 3 weeks during which the culture medium was replaced every 3 days. Cells were then fixed by $4 \%$ formalin (Sigma, USA) and washed by PBS. Osteocyte cell matrix was visible by alizarin red $(40 \mathrm{mM})$.

\section{Application of mechanical stimuli}

ASCs were exposed to cyclic shear stress $\left(0-2.5 \mathrm{dyn} / \mathrm{cm}^{2}\right.$, $1 \mathrm{~Hz})$ and cyclic stretch $(0-10 \%, 1 \mathrm{~Hz})$ for $24 \mathrm{~h}$ (Cevallos et al. 2006; Obi et al. 2012), while the SPA value was altered in three magnitudes of $0^{\circ},-45^{\circ}$ and $-90^{\circ}$. Such ranges of cyclic stretch and shear stress have been used among in vitro studies to simulate biological conditions (James et al. 1995; Nagel et al. 1994; Peng et al. 2000).

Cells were cultured on collagen coated silicon rubber membrane (PDMS) for $24 \mathrm{~h}$ and test samples were subjected to concurrent shear and tensile stresses with differing SPA values within the culture medium (15\% fetal bovine serum [FBS-(Gibco, USA)] low-glucose Dulbecco's modified Eagle's medium [DMEM-(Gibco, USA)] utilizing a custom made bioreactor. The control samples were cultured with the same conditions and kept for the same duration. After treatment, cells were transferred for further analyses.

To generate and apply pulsatile shear and circumferential stresses separately, a special custom-made bioreactor was utilized as previously described. Briefly, the device consisted of a cone and plate mechanism by which shear stress was applied on the cell seeded plate. ASCs were seeded on a collagen coated $(1 \mathrm{mg}$ collagen $/ \mathrm{mL}$ in $0.1 \%$ acetic acid, Sigma, USA) silicone membrane (Iran Polymer and Petrochemical Institute) and were exposed to cyclic shear stress of $0-2.5 \mathrm{dym} / \mathrm{cm}^{2}$. The magnitude of shear stress was achieved by a proper design of angle and dimensions of the cone, and considering culture medium as a Newtonian fluid with constant viscosity. Concurrently the membrane was subjected to $10 \%$ cyclic stretch through attachment to moving grippers (Fig. 1a). Two separate stepper motors (Delta electrics, Taiwan) provided the rotating movements of cone and oscillating movements of jaws. Shear stress has valued as high as $10-15 \mathrm{dyn} / \mathrm{cm}^{2}$ in some locations of vascular system near to the heart, but these locations generally experience in-phase shear stress and tensile stretches, and as a result, they are not theoretically vulnerable. However, the farther locations of the vascular system, which has been exposed to lower amount of shear stress, experience higher amounts of SPA, and therefore, they are more prone to some pathological conditions. Therefore, it has been tried to mimic these situations, and therefore, lower amount of pulsatile shear stresses have been utilized. A desired SPA was provided by calculated time delay between the rotation of cone and oscillation of jaws (Fig. 1b). 


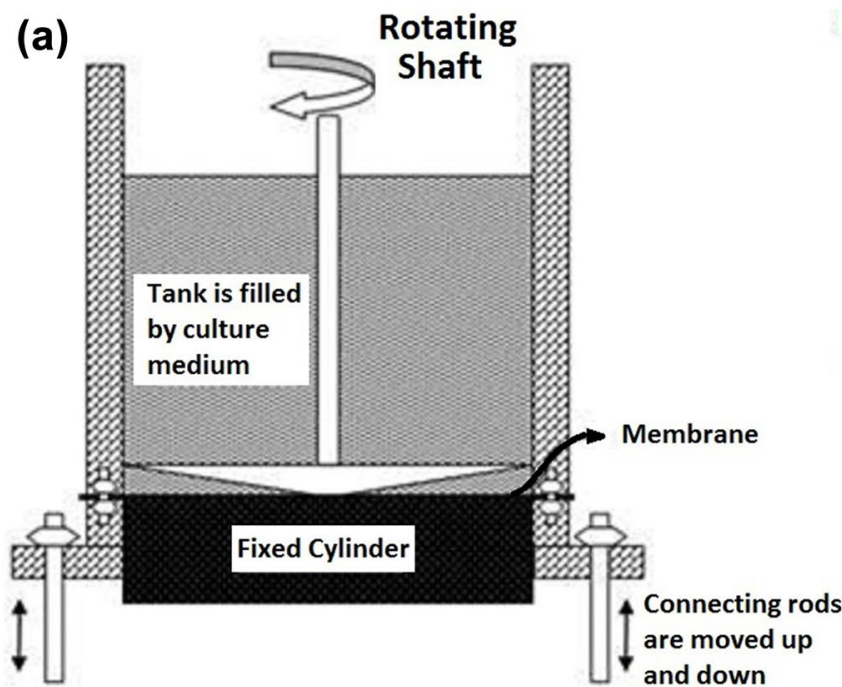

Fig. 1 a Schematic view of the bioreactor. Cells are cultured on a silicone membrane and a rheometer-like mechanism including a cone and plate system has been used to create shear stress. Cone has a slight angle which guarantees the uniform shear stress in different radii on substrate. Silicone membrane has been attached to bars that

\section{Gene expression}

For assessing the level of endothelial specific gene expression in ASCs among test and control samples, a real-time PCR assay was accomplished. Three independent experiments were performed in duplicate for real-time PCR. After application of mechanical forces on ASCs, the whole RNA was extracted by QIAGEN RNeasy plus mini kit (QIAGEN, USA) according to the manufacturer instructions and stored at $-80{ }^{\circ} \mathrm{C}$. The optical density of the RNA was determined and $1000 \mathrm{ng}$ of $R N A$ was reversely transcripted to cDNA by QuantiTect, QIAGEN Reverse Transcription Kit (QIAGEN, USA).

The expressions of three endothelial specific genes which are essential in the function of ECs were analyzed among control and test samples. FLK-1 is an essential endothelial marker involved in permeability, migration, proliferation, apoptosis, and vasodilation. VE-cadherin is mostly known as an endothelial specific intracellular adhesion protein which contributes to vascular permeability and provides adhesive structure for inner layer of blood vessels and is necessary for expansion and branching of vessels. Since this marker is responsible for proper adhesion among endothelial junctions, alteration in its level may lead to passage of unwanted large molecules such as lipids, leading to atherogenesis (Guo and Hamilton 1996; Suhalim et al. 2012). On the other hand, vWF is generally activated in endothelial injury. This marker is expressed exclusively by mature ECs mostly during pathological conditions specially thrombosis and plays vital role in (b)

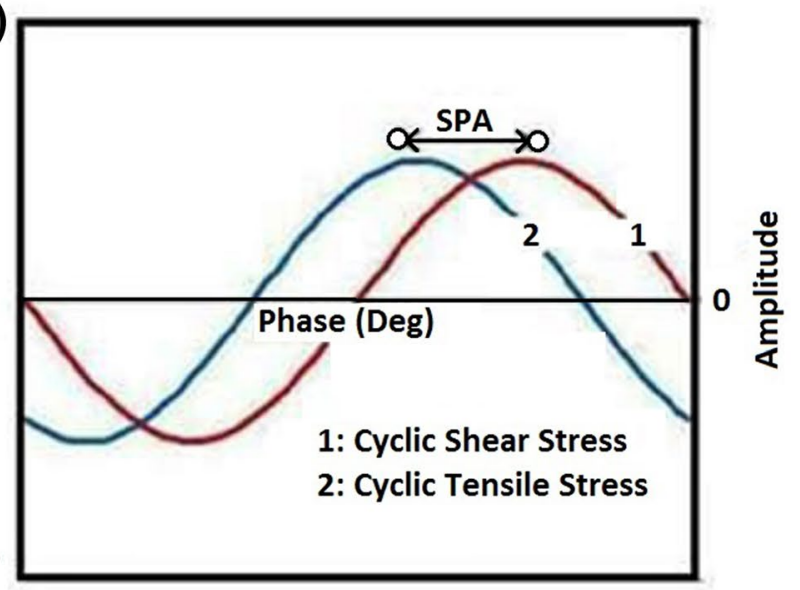

have the ability to move up and down to create this type of mechanical stretch movement. b Description of stress phase angle through cyclic shear and stretch stresses with normalized amplitudes. The time interval between rotating cone and oscillating jaws provides SPA

healing of endothelial injury through binding (Doggett et al. 2002).

SYBR green-based primers were designed by the Primer Express software (version 3) with the sequences as follows: house-keeping gene, glyceraldehyde 3-phosphate dehydrogenase (GAPDH), Forward, 5'-ACACCCACTCCTCCA CCTTTG- $3^{\prime}$ and reverse $5^{\prime}$-TCCACCACCCTGTTGCTG TAG-3'; FLK-1, forward, 5'-GACTTCCTGACCTTGGAG CATCT- $3^{\prime}$ and reverse 5'-GATTTTAACCACGTTCTT CTCCGA-3'; vWF, forward, 5'-TCTGTGGATTCAGTG GATGCA-3' and reverse 5'-CGTAGCGATCTCCAATTC CAA-3'; VE-cadherin, forward, 5'-TTTCCAGCAGCCTTT CTACCAC- $3^{\prime}$ and reverse $5^{\prime}$-GGAAGAACTGGCCCTTGT CAC-3'; $\alpha$-SMA, forward, 5'-ACACAACTGTGAATGTCC TGTGG-3', and reverse, 5'-GGTAACGAGTCAGAGCTT TGGC-3'; SMMHC, forward, 5'-GAGACGAGAGGACAT TCCACATC- $3^{\prime}$, and reverse, 5'-TTGGAGAGGAAGGTG TAGTTGTTG-3'.

\section{Cell elastic modulus}

To analyze elastic behavior of cell bodies atomic force microscopy (AFM) was used on cells after test duration for both test and control groups. Using Nanowizard 3 AFM device (JPK Instruments AG, Germany), the cantilever (CSC17/noAl, MikroMash), having nominal spring constant of $0.15 \mathrm{~N} / \mathrm{m}$, indented cells to apply $1 \mathrm{nN}$ force. The spring constant of each cantilever was measured using a single force measurement test over a glass substrate and applying thermal noise method (Hutter and Bechhoefer 1993). For 
each point of indentation, the force-indentation depth curve was obtained and the modified Hertz theory was fitted to measure the Young's modulus. According to tip shape of the used cantilever, the modified Hertz equation for conical shaped tips was implemented (Lin et al. 2007). In each test, 30 cells were randomly selected and indented in nearly 20 points over their nuclei.

\section{Actin fiber staining}

Due to its established role in mechano-transduction and providing biomechanical strength for cells, actin fiber structure of cells was studied among test and control samples. Actin staining was performed by Phalloidin (Sigma, USA) and scanned by a confocal microscope. Briefly, the culture medium was removed and cells washed with PBS several times. Then formaldehyde (Sigma, USA) in 3.7\% PBS was used for cell fixation followed by further washing. To make the membrane permeable to hue penetration, Triton X-100 $0.1 \%$ (Sigma, USA) in PBS was added for $10 \mathrm{~min}$. ASCs were washed again by PBS several times and $4 \mu \mathrm{g} / \mathrm{mL}$ phalloidin in PBS was added. The cells were then incubated at room temperature and protected from light for $45 \mathrm{~min}$. After further washing, cells were transferred for confocal microscopy.

\section{Results}

\section{Cell characterization}

Flow cytometry assay revealed that more than $95 \%$ of cells expressed the mesenchymal stem cell specific markers (CD44 and CD90), while they did not express hematopoietic stem cell markers (CD45) (Table 1).

\section{Multipotential assay}

Results of multipotential assay demonstrated that ASCs were capable of osteogenic and adipogenic differentiations. Alizarin red staining described generation of the osteocyte cell matrix among ASCs exposed to osteogenic differentiating medium and adipose vacuoles were visible when ASCs were exposed to adipogenic differentiating medium.

Table 1 Flow-cytometry results

\begin{tabular}{lclc}
\hline M1 (Isotype) & M2 & & MSCs \\
\hline 99.05 & 1.02 & CD44 & + \\
14.61 & 84.5 & CD90 & + \\
11.44 & 88.2 & CD45 & - \\
\hline
\end{tabular}

M1 is isotype and M2 describes CD90, CD44, and CD45

\section{Gene expression}

Real-time PCR assay indicated altered gene expression in ASCs when exposed to cyclic stresses with differing SPA values. Figure 2 compares the expression of endothelial specific genes among test groups with $\mathrm{SPA}=0,-45^{\circ}$, $-90^{\circ}$, and control group. In general, the combined loading with differing SPA values caused significant elevation of FLK-1 and VE-cadherin among each test group compared to control group $(P<0.05)$. Application of combined cyclic stresses with SPA $=0$ significantly elevated two major endothelial specific genes of FLK-1 and VEcadherin by 1.43 and 22.47 -folds $(P<0.05)$ (Fig. 2 d). On the other hand, this loading regime did not cause any significant changes in the expression level of vWF $(P>0.05)$ (Fig. 2d).

Results demonstrated significant decrease of nearly 35 and $73 \%$ in the expression of FLK-1 when SPA reached $-45^{\circ}$ and $-90^{\circ}$ from $0^{\circ}$, respectively $(P<0.0 .5)$. For VEcadherin marker, such reductions were accordingly 32 and $65 \%(P<0.0 .5)$. The trend for vWF expression was reverse. When SPA reached the values of $-45^{\circ}$ and $-90^{\circ}$ from base line of 0 , the expression levels of vWF increased by 44 and $127 \%$ correspondingly $(P<0.0 .5)$. When alterations in the gene expression level were compared among all the test and control groups through ANOVA analysis, results indicated that regardless of increase or decrease, alterations were statistically significant for all three genes $(P<0.05)$.

\section{Cell elastic modulus}

Alterations in Young's modulus of ASCs when exposed to concurrent loadings with differing SPA are shown Fig. 3. The results indicated significant stiffening of cells for each test group compared to control samples $(P<0.05)$. The average increase of Young's modulus of ASCs compared to that of control group was $159 \%$ for samples exposed to SPA $=0$, $123 \%$ for samples subjected to SPA $=-45^{\circ}$ and $89 \%$ among samples exposed to SPA $=-90^{\circ}$ (Fig. 3). When all test and control groups were compared through ANOVA analysis, cell stiffening was found to be significant $(P<0.05)$.

\section{Actin fibers staining}

Phalloidin staining of actin fibers was carried out for test and control groups. Figure 4 shows samples of confocal microscope imaging of stained actin fibers among four groups. Results described that application of combined mechanical loadings had determinant effects on actin fiber structure and arrangement of cells describing more vivid and thicker fibers among samples treated mechanically in comparison to control samples. Compared to ASCs without any treatment, samples subjected to combined cyclic shear-tension 


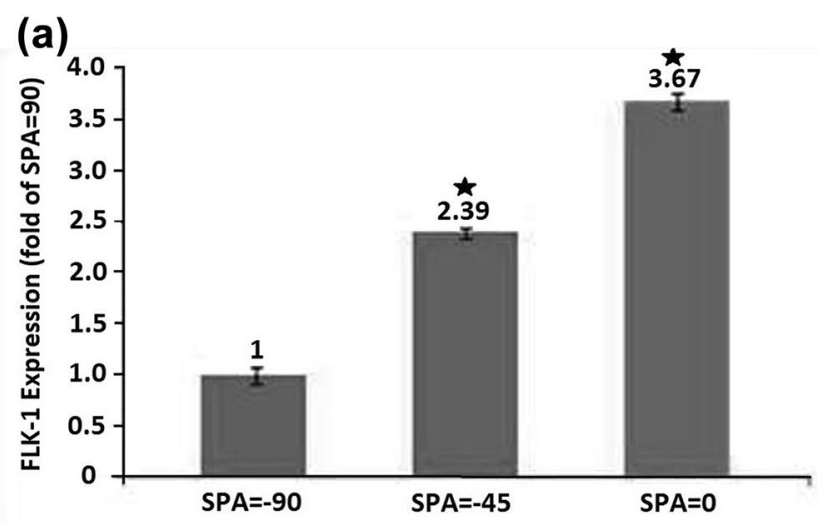

(b)
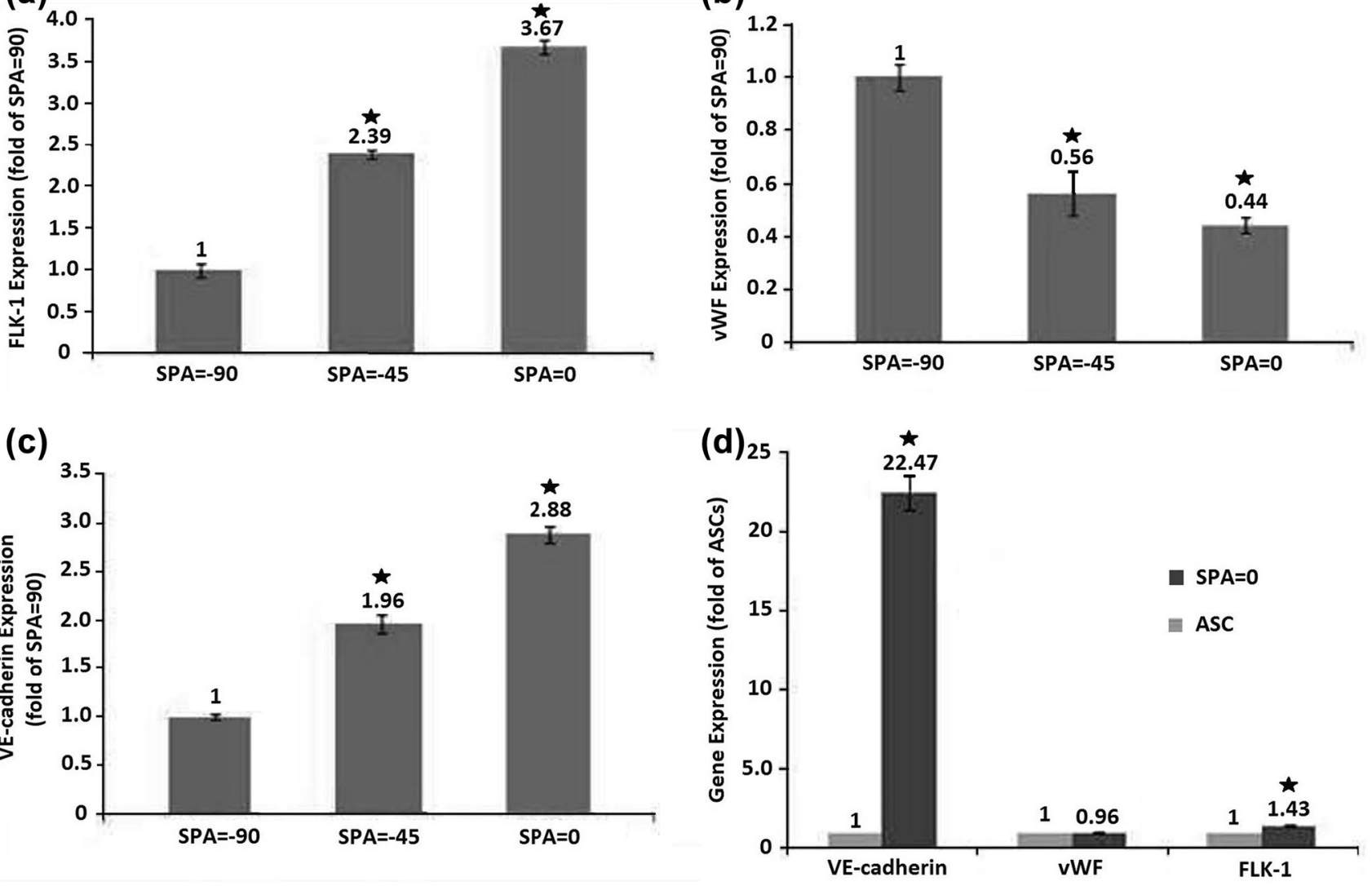

Fig. 2 Expression of FLK-1 (a), vWF (b) and VE-cadherin (c) in ASCs, exposed to SPA $=0,-45^{\circ}$ and $-90^{\circ}$. $\mathbf{d}$ is for the comparison of SPA $=0$ and ASCs as control group. The error bars indicate

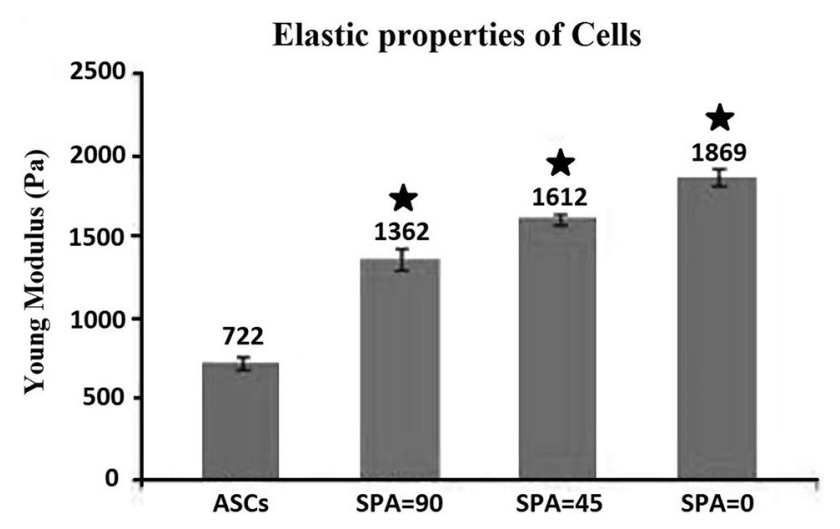

Fig. 3 Young's modulus of ASCs among control samples and samples exposed to combined tensile and shear stresses with SPA $=0$, $-45^{\circ}$, and $-90^{\circ}$. Error bars show standard deviation and stars show significant difference when each test group was compared to control group $(P<0.05)$

developed distinct fibrous structure through stress fiber generation and organization (Fig. 4b-d). Among test groups, samples with SPA $=0$ indicated the thickest fibers and

standard deviation and stars show statistical significant difference $(P<0.05)$. Elevation of SPA had negative effects on the expressions of endothelial specific genes in ASCs

highest generation of stress fibers (Fig. 4d). ASCs without any treatment (control samples) showed poor organized actin structure.

\section{Discussion}

It is well established that biomechanical environment of ECs regulates endothelial vital function within the cardiovasculature (Li et al. 2005). Among arteries cyclic wall shear stress that is due to blood flow accompanied by cyclic circumferential stress caused by pulsatile blood pressure, as two main hemodynamic parameters, determine endothelial function and maintain the homeostatic state of arteries. A parameter range outside biological condition may cause endothelial injury which is the main source of human mortality (Tada et al. 2007). Moreover, the temporal phase angle between these pulses, has been emphasized to play highlighted role in endothelial behavior (Qiu and Tarbell 2000a). It has been hypothesized that the value of stress phase angel (SPA) is a determinant of endothelial behavior. Cyclic shear and circumferential stresses are often markedly out of phase in the 

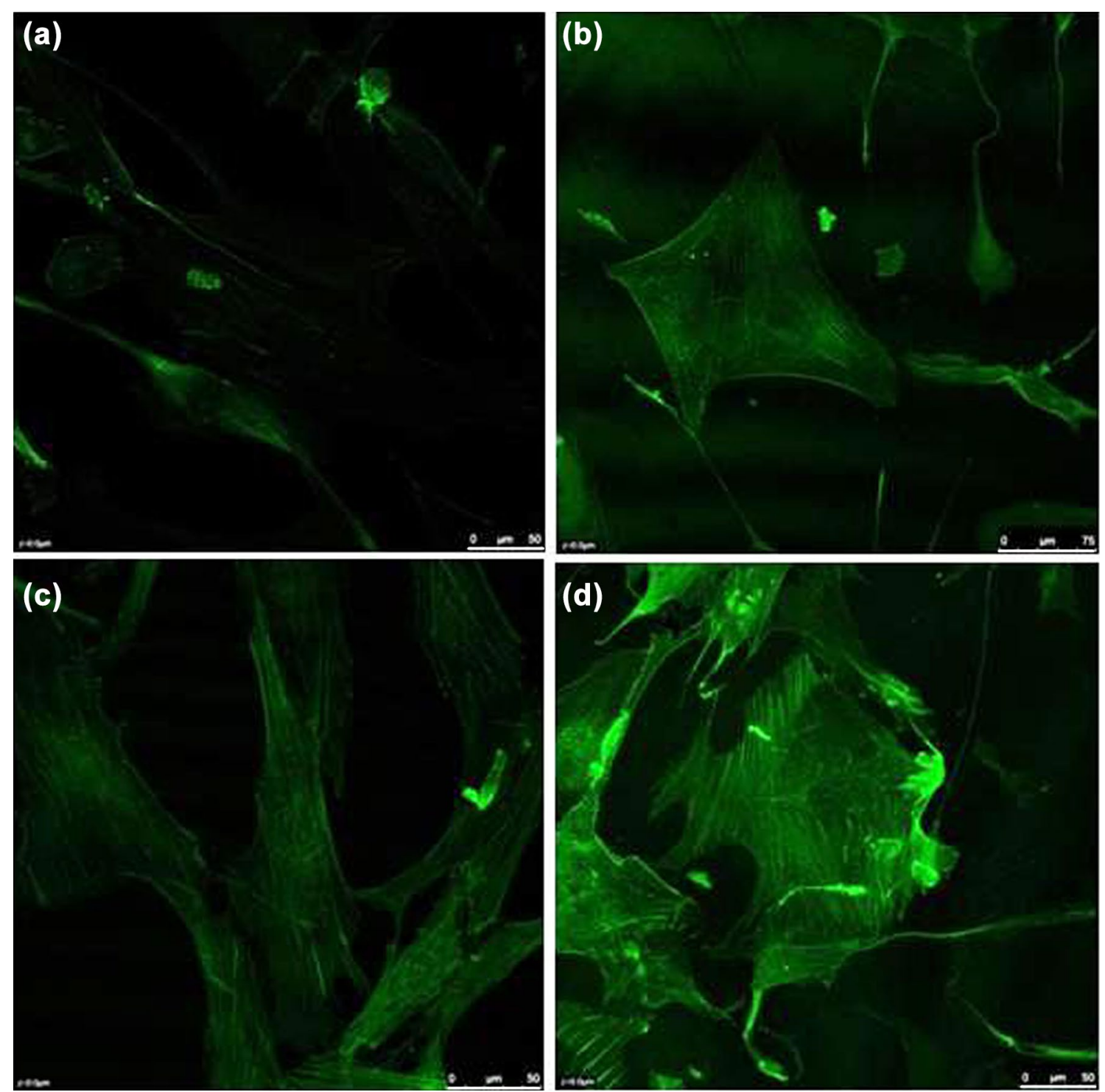

Fig. 4 Confocal imaging of phalloidin staining of actin fibers for a ASCs without any biomechanical treatment, b-d ASCs exposed to combined cyclic shear and tensile stresses with SPA $=-90^{\circ}, \mathrm{SPA}=-45^{\circ}$ and SPA $=0$, respectively

vulnerable regions of plaque formation and atherosclerotic arteries (Qiu and Tarbell 2000a; Tada and Tarbell 2005).

Here, we hypothesized that SPA is an influential parameter in differentiation of mesenchymal stem cells toward endothelial phenotype. This might give clue in achieving functional engineered ECs for therapeutical applications and indicate insight into mechanisms by which endothelial injury occurs in pathological conditions. We conducted experiments that assessed effects of differing SPA on behavior of ASCs through analysis of gene expression, elastic properties of cells, and actin cytoskeleton structure.

Results indicated that application of out-of-phase SPA resulted in lesser expression of vital endothelial genes and softer cell body with a diverse actin fiber skeleton. In other words, more negative SPA values have lagged the endothelial differentiation of ASCs. Among three vital endothelial genes, the expression level of vWF was less prominent, that is the only marker among three markers which is expressed more during pathologic conditions dealt with endothelial injury. The results are consistent with the idea that higher absolute values of SPA are correlated with dysfunctioning ECs.

FLK1 and VE-cadherin are two major markers essential in endothelial behavior. They are not only regulators of biological function of ECs, but also play major roles in mechano-transduction of environmental stimuli among which hemodynamic features are determinants in functionality of ECs within vasculature. A marked upregulation of 
VE-cadherin (up to 22.47-folds for in-phase shear-tension waves) was observed when ASCs were subjected to concurrent cyclic shear-tensile loadings. This confirms that a micromechanical environment similar to that of functional ECs within cardiovascular system assists endothelial differentiation of ASCs. However, this depends on the phase difference between two mechanical stimuli. In addition to permeability, migration and proliferation of ECs (Holmes et al. 2007), FLK-1, as an endothelial specific marker, determines strain energy density of membrane which directly affects the opening of ion channels and eventually regulates cell permeability (Sachs 2010). In current study the expression of FLK-1 in samples with SPA $=-90^{\circ}$ was only $27 \%$ of those of SPA $=0$.

Compared to VE-cadherin, a modest, yet statistically significant upregulation of FLK-1 (up to 1.43-folds for in-phase shear-tension waves) was observed. It has been demonstrated that VE-cadherin actively contributes to the control of endothelial cohesion and intracellular junctions (Ohashi et al. 2007). The interaction of blood with the surrounding tissues is mainly regulated by the endothelium. The appropriate function of VE-cadherin and claudin-5 that are the key elements in adherence and tight endothelial junctions would be determinant of the barrier properties of endothelium to unwanted macromolecules which induce atherogenesis. In some serious pathological conditions among arteries, abnormal increase of vascular permeability has shown to be evident (Gavard 2009). The loss of VE-cadherin occurs due to the permeability inducing factors (PIFs) which dismantle VE-cadherin functionality by phosphorylation, internalization, and catenin dissociation (Gavard 2009). In current study, the expression level of VE-cadherin of samples exposed to $\mathrm{SPA}=-90^{\circ}$ was only $35 \%$ of those exposed to $\mathrm{SPA}=0$.

The function of Willebrand factor ( $\mathrm{vWF}$ ) is rather different from other two genes, since it is related to injury conditions. The vWF marker plays an important role in platelet adhesion along the wound sites within the arteries (Sadler 1998; Wu et al. 2008). Past studies have explained that the platelet aggregation and adhesion is regulated by release of vWF (Vischer 2006), and subsequently, the high level of vWF has been attributed indirectly to atherosclerosis and thrombogenesis (Lip and Blann 1997; Vischer 2006). Hence, it is not surprising that results of our research describes signified higher values of vWF as the SPA reaches higher negative values, since higher SPA values have been shown to be associated with atherosclerosis and thrombogenesis (Qiu and Tarbell 2000a). The average vWF level was 1.78 and 2.27 higher corresponding to test groups with $\mathrm{SPA}=-45^{\circ}$ and $\mathrm{SPA}=-90^{\circ}$ compared to samples with $\mathrm{SPA}=0$.

The differentiation of ASCs was accompanied by cytoskeletal development through enhancement of actin fiber structure and generation and bundling of new fibers which results in stiffening of the cell body as measured in current study. However, such effect was weakened when cells were treated by more negative SPA values. The arrangement and organization of cytoskeleton are the determinant factor in Young's modulus of cell bodies. The elastic modulus of cells is related to the content and arrangement of cytoskeletal elements especially actin fibers. Since cell stiffness is influenced by the culture conditions and substrate properties (Byfield et al. 2009), a wide range of 2000-7000 Pa has been suggested for matured human aortic endothelial cells and human umbilical vein endothelial cells (Mathur et al. 2001). Our results suggest that ASCs treated by synchronic phase of shear and circumferential stresses $(\mathrm{SPA}=0)$ has the closest value of elastic modulus to that of normal mature ECs. It has been well demonstrated that function of endothelial cells is related to the elastic property of the cell body and cytoskeletal arrangement (Mathur et al. 2001). In some clinical conditions, the stiffness of ECs was shown to be reduced (Lekka et al. 2012). Highly deformable ECs are more prone to passage of large molecules (especially in atherogenesis) and other cells through endothelium (Simoneau et al. 2012). Such phenomenon is also of great importance in cancer development in which cancerous cells pass through endothelial junctions during metastasis state (Simoneau et al. 2012).

Cell stiffness is determined by the thickness and arrangement of bundles of actin fibers. Application of biomechanical stimuli results in remodeling of the cell body mainly through alignment and generation of stress fibers (Wang et al. 2001). In this study, the effects of cyclic shear and circumferential stresses have been emphasized on both cell elasticity and actin structure. Cells under mechanical stimuli were shown to be stiffer and have more actin content than ASCs (Figs. 3, 4). Moreover, the phase difference between shear and tensile stresses was shown to be significantly effective. When the phase difference was smaller, the cell body was stiffer and the actin structure became richer. It is likely that application of smaller phase difference on ASCs during differentiation results in cells with characteristics more similar to functional ECs. While in all biomechanically motivated groups, actin fibers were semi-organized suggested to be in the direction of minimal substrate deformation (Wang et al. 2001), the fibers in group of SPA $=0$ were shown to be thicker and more organized compared to other groups of SPA $=-90^{\circ}$ and $\mathrm{SPA}=-45^{\circ}$ (Fig. 4). It can be implied that cell stiffening was mostly due to enhanced cytoskeleton. The shear stress or tensile stretch alone cannot perfectly and completely mimic the inherent unique mechanical environment of endothelial cells and this fact is even more highlighted when we understand that shear stress or tensile stretch alone have been used on other cell phenotypes as a motivator for differentiation of mesenchymal stem cells. For instance, the uniaxial and equiaxial tensile stretches 
alone have been widely used for osteogenic (Qi et al. 2008) and cardiomyocyte (Huang et al. 2012) differentiation of stem cells and our investigations showed that not only for endothelial differentiation, but also shear stress alone has been utilized for chondrogenic (Alves da Silva et al. 2011) and osteogenic (Yourek et al. 2010) differentiation of different colonies of mesenchymal stem cells. The idea behind these applications is that, for example, shear stress alone does exist in many in vivo biological environments such as articular joints, as the synovial fluid flows during movement, and therefore, shear stress alone can induce chondrogenic differentiation or a shear stress that is created in trabecular bone which performs as a stimulator of osteoblasts and osteoclasts. Even shear stress alone has been used for cardiomyogenic differentiation (Huang et al. 2010). However, the combination of shear stress and tensile stretch can be regarded as a unique mechanical environment special for blood vessels and logically their results have more relative significance.

\section{Conclusion}

Beside the independent role of biomechanical forces on cell behavior, their interaction may also influence cell behavior. In this study, the endothelial differentiation of ASCs was shown to be influenced by shear/tensile stresses and their interaction characterized by SPA. In was shown that application of in-phase SPA incites conditions related to healthy ECs, while larger phase differences were related to dysfunctioning ECs. This may provide some clues for determination of pathophysiological mechanism that lies under endothelial injury and atherogenesis. In addition, the results assist in determination of proper mechanical stimuli for functional endothelial differentiation of mesenchymal stem cells.

Acknowledgements Authors would like to thank Dr. Mahdian, Dr. Amanzadeh from Pasteur Institute of Iran, whose contributions are memorable.

Funding This study was not funded by any company or Grant.

\section{Compliance with ethical standards}

Conflict of interest Author Shahrokh Shojaei declares that he has no conflict of interest. Author Mohammad Tafazzoli-Shadpour declares that he has no conflict of interest. Author Nooshin Haghighipour declares that she has no conflict of interest. Author Mohammad-Ali Shokrgozar declares that he has no conflict of interest. Author Fatemeh Hejazi Jahromi declares that she has no conflict of interest.

Research involving human participants and/or animals All procedures performed in studies involving human participants were in accordance with the ethical standards of the institutional and/or national research committee and with the 1964 Helsinki declaration and its later amendments or comparable ethical standards.

Informed consent Informed consent was obtained from all individual participants included in the study.

Open Access This article is distributed under the terms of the Creative Commons Attribution 4.0 International License (http://creativeco mmons.org/licenses/by/4.0/), which permits unrestricted use, distribution, and reproduction in any medium, provided you give appropriate credit to the original author(s) and the source, provide a link to the Creative Commons license, and indicate if changes were made.

\section{References}

Alves da Silva ML, Martins A, Costa-Pinto A, Correlo V, Sol P, Bhattacharya M, Neves N (2011) Chondrogenic differentiation of human bone marrow mesenchymal stem cells in chitosan-based scaffolds using a flow-perfusion bioreactor. J Tissue Eng Regen Med 5(9):722-732

Barron V, Brougham C, Coghlan K, McLucas E, O’Mahoney D, Stenson-Cox C, McHugh PE (2007) The effect of physiological cyclic stretch on the cell morphology, cell orientation and protein expression of endothelial cells. J Mater Sci Mater Med 18(10):1973-1981

Bassiouny HS, White S, Glagov S, Choi E, Giddens DP, Zarins CK (1992) Anastomotic intimal hyperplasia: mechanical injury or flow induced. J Vasc Surg 15(4):708-717

Byfield FJ, Reen RK, Shentu T-P, Levitan I, Gooch KJ (2009) Endothelial actin and cell stiffness is modulated by substrate stiffness in 2D and 3D. J Biomech 42(8):1114-1119

Cevallos M, Riha GM, Wang X, Yang H, Yan S, Li M, Chen C (2006) Cyclic strain induces expression of specific smooth muscle cell markers in human endothelial cells. Differentiation 74(9):552-561

Charoenpanich A, Wall ME, Tucker CJ, Andrews DM, Lalush DS, Dirschl DR, Loboa EG (2014) Cyclic tensile strain enhances osteogenesis and angiogenesis in mesenchymal stem cells from osteoporotic donors. Tissue Eng Part A. 20(1-2):67-78. https:// doi.org/10.1089/ten.TEA.2013.0006

Charrier EE, Pogoda K, Wells RG, Janmey PA (2018) Control of cell morphology and differentiation by substrates with independently tunable elasticity and viscous dissipation. Nat Commun 9(1):449

Dancu MB, Tarbell JM (2006) Large negative stress phase angle (SPA) attenuates nitric oxide production in bovine aortic endothelial cells. J Biomech Eng 128(3):329

Doggett TA, Girdhar G, Lawshé A, Schmidtke DW, Laurenzi IJ, Diamond SL, Diacovo TG (2002) Selectin-like kinetics and biomechanics promote rapid platelet adhesion in flow: the GPIb $\alpha$-vWF Tether bond. Biophys J 83(1):194-205

Dolan JM, Meng H, Singh S, Paluch R, Kolega J (2011) High fluid shear stress and spatial shear stress gradients affect endothelial proliferation, survival, and alignment. Ann Biomed Eng 39(6):1620-1631

Elhadj S, Akers RM, Forsten-Williams K (2003) Chronic pulsatile shear stress alters insulin-like growth factor-I (IGF-I) binding protein release in vitro. Ann Biomed Eng 31(2):163-170

Estes BT, Diekman BO, Gimble JM, Guilak F (2010) Isolation of adipose-derived stem cells and their induction to a chondrogenic phenotype. Nat Protoc 5(7):1294-1311

Gavard J (2009) Breaking the VE-cadherin bonds. FEBS Lett 583(1):1-6 
Guo W, Hamilton JA (1996) 13C MAS NMR studies of crystalline cholesterol and lipid mixtures modeling atherosclerotic plaques. Biophys J 71(5):2857-2868

Haga JH, Li Y-SJ, Chien S (2007) Molecular basis of the effects of mechanical stretch on vascular smooth muscle cells. J Biomech 40(5):947-960

Haghighipour N, Tafazzoli-Shadpour M, Shokrgozar MA, Amini S (2010) Effects of cyclic stretch waveform on endothelial cell morphology using fractal analysis. Artif Org 34(6):481-490

Holmes K, Roberts OL, Thomas AM, Cross MJ (2007) Vascular endothelial growth factor receptor-2: structure, function, intracellular signalling and therapeutic inhibition. Cell Signal 19(10):2003-2012

Huang Y, Jia X, Bai K, Gong X, Fan Y (2010) Effect of fluid shear stress on cardiomyogenic differentiation of rat bone marrow mesenchymal stem cells. Arch Med Res 41(7):497-505

Huang Y, Zheng L, Gong X, Jia X, Song W, Liu M, Fan Y (2012) Effect of cyclic strain on cardiomyogenic differentiation of rat bone marrow derived mesenchymal stem cells. PLoS One 7(4):e34960

Hutter JL, Bechhoefer J (1993) Calibration of atomic-force microscope tips. Rev Sci Instrum 64(7):1868-1873

James N, Harrison D, Nerem R (1995) Effects of shear on endothelial cell calcium in the presence and absence of ATP. FASEB J 9(10):968-973

Jeon H, Simon CG, Kim G (2014) A mini-review: cell response to microscale, nanoscale, and hierarchical patterning of surface structure. J Biomed Mater Res B Appl Biomater 102(7):1580-1594

Joshi AK, Leask RL, Myers JG, Ojha M, Butany J, Ethier CR (2004) Intimal thickness is not associated with wall shear stress patterns in the human right coronary artery. Arterioscler Thromb Vasc Biol 24(12):2408-2413

Kang H, Cancel LM, Tarbell JM (2014) Effect of shear stress on water and LDL transport through cultured endothelial cell monolayers. Atherosclerosis 233(2):682-690

Ku DN, Giddens DP, Zarins CK, Glagov S (1985) Pulsatile flow and atherosclerosis in the human carotid bifurcation. Positive correlation between plaque location and low oscillating shear stress. Arterioscler Thromb Vasc Biol 5(3):293-302

La A, Tranquillo RT (2018) Shear conditioning of adipose stem cells for reduced platelet binding to engineered vascular grafts. Tissue Eng Part A. https://doi.org/10.1089/ten.tea.2017.0475

Lekka M, Gil D, Pogoda K, Dulińska-Litewka J, Jach R, Gostek J, Klymenko O, Prauzner-Bechcicki S, Stachura Z, Wiltowska-Zuber J, Okoń K, Laidler P (2012) Cancer cell detection in tissue sections using AFM. Arch Biochem Biophys. 518(2):151-156. https://doi. org/10.1016/j.abb.2011.12.013

Li Y-SJ, Haga JH, Chien S (2005) Molecular basis of the effects of shear stress on vascular endothelial cells. J Biomech 38(10):1949-1971

Lin DC, Dimitriadis EK, Horkay F (2007) Robust strategies for automated AFM force curve analysis-I. Non-adhesive indentation of soft, inhomogeneous materials. J Biomech Eng 129(3):430-440

Lip GY, Blann A (1997) von Willebrand factor: a marker of endothelial dysfunction in vascular disorders? Cardiovasc Res 34(2):255-265

Mathur AB, Collinsworth AM, Reichert WM, Kraus WE, Truskey GA (2001) Endothelial, cardiac muscle and skeletal muscle exhibit different viscous and elastic properties as determined by atomic force microscopy. J Biomech 34(12):1545-1553

Maul TM, Chew DW, Nieponice A, Vorp DA (2011) Mechanical stimuli differentially control stem cell behavior: morphology, proliferation, and differentiation. Biomech Model Mechanobiol 10(6):939-953

Nagel T, Resnick N, Atkinson WJ, Dewey CF Jr, Gimbrone MA Jr (1994) Shear stress selectively upregulates intercellular adhesion molecule-1 expression in cultured human vascular endothelial cells. J Clin Invest 94(2):885

Obi S, Masuda H, Shizuno T, Sato A, Yamamoto K, Ando J, Asahara $\mathrm{T}$ (2012) Fluid shear stress induces differentiation of circulating phenotype endothelial progenitor cells. Am J Physiol Cell Physiol 303(6):C595-C606

Ohashi T, Sugaya Y, Sakamoto N, Sato M (2007) Hydrostatic pressure influences morphology and expression of VE-cadherin of vascular endothelial cells. J Biomech 40(11):2399-2405

Owatverot TB, Oswald SJ, Chen Y, Wille JJ, Yin FC (2005) Effect of combined cyclic stretch and fluid shear stress on endothelial cell morphological responses. J Biomech Eng 127(3):374

Paim A, Braghirolli D, Cardozo N, Pranke P, Tessaro I (2018) Human dental pulp stem cell adhesion and detachment in polycaprolactone electrospun scaffolds under direct perfusion. Braz J Med Biol Res 51(5):e6754

Paul NE, Denecke B, Kim BS, Dreser A, Bernhagen J, Pallua N (2018) The effect of mechanical stress on the proliferation, adipogenic differentiation and gene expression of human adipose-derived stem cells. J Tissue Eng Regen Med 12(1):276-284

Peng X, Recchia FA, Byrne BJ, Wittstein IS, Ziegelstein RC, Kass DA (2000) In vitro system to study realistic pulsatile flow and stretch signaling in cultured vascular cells. Am J Physiol Cell Physiol 279(3):C797-C805

Qi M-C, Hu J, Zou SJ, Chen HQ, Zhou HX, Han LC (2008) Mechanical strain induces osteogenic differentiation: Cbfa1 and Ets-1 expression in stretched rat mesenchymal stem cells. Int J Oral Maxillofac Surg 37(5):453-458

Qiu Y, Tarbell JM (2000a) Interaction between wall shear stress and circumferential strain affects endothelial cell biochemical production. J Vasc Res 37(3):147-157

Qiu Y, Tarbell JM (2000b) Numerical simulation of pulsatile flow in a compliant curved tube model of a coronary artery. J Biomech Eng 122(1):77-85

Sachs F (2010) Stretch-activated ion channels: what are they? Physiology 25(1):50-56

Sadler JE (1998) Biochemistry and genetics of von Willebrand factor. Annu Rev Biochem 67(1):395-424

Shoajei S, Tafazzoli-Shahdpour M, Shokrgozar MA, Haghighipour N (2014) Alteration of human umbilical vein endothelial cell gene expression in different biomechanical environments. Cell Biol Int 38(5):577-581. https://doi.org/10.1002/cbin.10237

Shojaei S, Farokhi M, Omidvar R, Mottaghitalab F, Haghighipour N, Shokrgozar M, Ai J (2013a) Essential functionality of endometrial and adipose stem cells in normal and mechanically motivated conditions. J Biomater Tissue Eng 3(5):581-588

Shojaei S, Tafazzoli-Shahdpour M, Shokrgozar MA, Haghighipour N (2013b) Effects of mechanical and chemical stimuli on differentiation of human adipose-derived stem cells into endothelial cells. Int J Artif Org 36(9):663-673

Shojaei S, Tafazzoli-Shahdpour M, Shokrgozar MA, Haghighipour N (2013c) Effects of mechanical and chemical stimuli on differentiation of human adipose-derived stem cells into endothelial cells. Int J Artif Org 36(9):663-673

Shojaei S, Tafazzoli-Shahdpour M, Shokrgozar MA, Haghighipour $\mathrm{N}$ (2015) Comparative analysis of effects of cyclic uniaxial and equiaxial stretches on gene expression of human umbilical vein endothelial cells. Cell Biol Int 39(6):741-749

Simoneau B, Houle F, Huot J (2012) Regulation of endothelial permeability and transendothelial migration of cancer cells by tropomyosin-1 phosphorylation. Vasc Cell 4(1):18

Steinman DA, Thomas JB, Ladak HM, Milner JS, Rutt BK, Spence JD (2002) Reconstruction of carotid bifurcation hemodynamics and wall thickness using computational fluid dynamics and MRI. Magn Reson Med 47(1):149-159 
Suhalim JL, Chung C-Y, Lilledahl MB, Lim RS, Levi M, Tromberg BJ, Potma EO (2012) Characterization of cholesterol crystals in atherosclerotic plaques using stimulated Raman scattering and secondharmonic generation microscopy. Biophys J 102(8):1988-1995

Tada S, Tarbell J (2005) A computational study of flow in a compliant carotid bifurcation-stress phase angle correlation with shear stress. Ann Biomed Eng 33(9):1202-1212

Tada S, Dong C, Tarbell JM (2007) Effect of the stress phase angle on the strain energy density of the endothelial plasma membrane. Biophys J 93(9):3026-3033

Vining KH, Mooney DJ (2017) Mechanical forces direct stem cell behaviour in development and regeneration. Nat Rev Mol Cell Biol 18(12):728

Vischer U (2006) von Willebrand factor, endothelial dysfunction, and cardiovascular disease. J Thromb Haemost 4(6):1186-1193

Wang JH-C, Goldschmidt-Clermont P, Wille J, Yin FC-P (2001) Specificity of endothelial cell reorientation in response to cyclic mechanical stretching. J Biomech 34(12):1563-1572
Wu C-C, Chao Y-C, Chen C-N, Chien S, Chen Y-C, Chien C-C, Linju BL (2008) Synergism of biochemical and mechanical stimuli in the differentiation of human placenta-derived multipotent cells into endothelial cells. J Biomech 41(4):813-821

Yamamoto K, Takahashi T, Asahara T, Ohura N, Sokabe T, Kamiya A, Ando J (2003) Proliferation, differentiation, and tube formation by endothelial progenitor cells in response to shear stress. J Appl Physiol 95(5):2081-2088

Yourek G, McCormick SM, Mao JJ, Reilly GC (2010) Shear stress induces osteogenic differentiation of human mesenchymal stem cells. Regen Med 5(5):713-724

Publisher's Note Springer Nature remains neutral with regard to jurisdictional claims in published maps and institutional affiliations. 